\title{
EN BUSCA DE HUELLAS PARA LA RELACIÓN CIUDAD-COMUNICACIÓN Y EDUCACIÓN
}

\author{
Mónica Cuervo Prados*
}

\section{Resumen}

Este artículo presenta los resultados de la investigación denominada "Estudio Teórico sobre Comunicación y Ciudadanía", desarrollado desde la Línea de Comunicación y Ciudadanía de la Facultad de Comunicación Social de la Universidad Santo Tomás.

\section{Palabras clave}

Ciudad, comunicación - educación, ciudadanía.

\section{Abstract}

This article introduces the results presented by the research called "Theoretical Study about Communication and citizenship", developed from the Communication and Citizenship Line of the Social Communication Faculty of The Santo Tomás University.

\section{Key words}

City, communication - education, citizenship.

Comunicadora Social con Énfasis en Comunicación Educativa de la Pontificia Universidad Javeriana. Magíster en Comunicación de la Pontificia Universidad Javeriana. Decana de la Facultad de Comunicación Social y Directora Grupo Investigación Comunicación Paz/Conflicto y Línea Comunicación y Ciudadanía, Universidad Santo Tomás. Directora Grupo Procesos Comunicativos en el Ámbito Educativo. 
Dentro de la línea de Comunicación y Ciudadanía, del Grupo de Investigación de Comunicación, Paz y Conflicto de la Facultad de Comunicación Social de la Universidad Santo Tomás, se desarrolló una investigación documental-teórica de orden exploratorio, la cual se centró en la revisión de varios documentos sobre los distintos movimientos emprendidos por las ciencias sociales para comprender la ciudad como lugar educativo para el ciudadano.

Este estudio se generó como un aporte al documento de la línea de investigación mencionada y se enmarcó en el problema de investigación icuáles son los referentes teóricos que han aportado a la relación comunicación-educación y ciudadanía?, y en una sistematización del problema que tomó en cuenta los autores, enfoques teóricos, lineamientos y aproximaciones metodológicas. Para la recolección y sistematización de la información se utilizó una matriz de orden transversal, la cual retomó la sistematización del problema.

Dentro de los resultados obtenidos, se pudo observar que dentro de los autores que trabajan esta temática, se encuentran Lynch, Lorenzo, Tonucci, Gandinno, Manuetti, Tonnucci, Moncada, Trillas, Páramo, Álvarez, Noguera y Gadotti. Asimismo, se pudo establecer que el enfoque teórico más encontrado, desde los autores anteriores, es el del Movimiento Ciudad Educadora (Barcelona, 1990), el cual permea tanto el enfoque sobre "La Ciudad como Espacio Educativo" (Noguera, Álvarez y Castro, 2000), como los lineamientos sobre "Ciudadescuela-escuela-ciudad" que están establecidos desde el Plan Sectorial de educación para Bogotá (2004-2008).

En ese orden de ideas, se realizó específicamente la selección de los autores que aportaban más al enfoque del Movimiento Ciudad Educadora. Al cruzar la información obtenida, se focalizó el trabajo en Moncada, Trillas, Álvarez, Noguera y Gadotti.

Dentro de esta última fase del procedimiento, se encontró al respecto que "el movimiento de Ciudad Educadoraha favorecido la idea de que toda la ciudad pueda organizarse para promover patrones de oportunida- des de aprendizaje. La ciudad puede planearse como un lugar diseñado a propósito para el aprendizaje a lo largo de toda la vida y para el crecimiento personal" (Cuervo y Páramo, 2005).

De igual manera, se pudo aclarar que el concepto de ciudad educadora surge del Documento de la UNESCO "Aprendiendo a Ser" (Faure, 1972), el cual es ampliado desde el Encuentro de Ciudad Educadora realizado en 1990 en Barcelona. Este movimiento se centra, desde los encuentros de las personas y la participación ciudadana, en la recuperación de los ambientes de la ciudad como recursos pedagógicos, en una ciudad que debe reconocer su historia, su comunicación, sus reglas de apropiación y sus propuestas de desarrollo e identidad.

De acuerdo con este planteamiento, una ciudad será educadora cuando sus instituciones puedan reconocer, ejercitar y desarrollar una función educadora en adición a sus funciones económicas, sociales y políticas, y al suministro de servicios" (Barcelona, 1990). "Una ciudad educadora debería aceptar como fin y responsabilidad la educación, avance y desarrollo de todos sus habitantes (Cuervo y Páramo, 2005).

Se pudo entonces resaltar que, "el concepto de Ciudad Educadora es un concepto que enfatiza lo público y colectivo, lo político y ético. Usa la educación como un medio principalmente comunicativo que empodera la sociedad sobre su propio destino, cambiando las conductas y reforzando la democracia a través de la participación ciudadana. En la actualidad, y siguiendo la agenda de la Asociación Internacional de Ciudades Educadoras, es posible identificar seis líneas de trabajo, a saber: "la apropiación del espacio a través del esfuerzo de los ciudadanos; el desarrollo local; la educación; el empleo y el ocio; la escuela y nuevos modelos de educación ciudadana y la memoria e identidad con la ciudad" (Cuervo y Páramo, 2005).

Es importante resaltar que en nuestro país este movimiento ha tenido ingreso con el apoyo de la Organización de los Estados Iberoamericanos, OEI. Este aporte 
ha iniciado una mirada en la cual incluso la comunicación-cultura política se ha incluido, al ver al sujeto social en un contexto plagado de procesos comunicativos ligados a matrices culturales, formatos industriales, lógicas de producción y competencias de recepción, que desde mediaciones como la institucionalidad, la ritualidad, la tecnicidad y la socialidad, dan una responsabilidad esencial a la relación entre comunicación y educación.

Específicamente en Bogotá, esta mirada teórica a nivel administrativo (Alcaldía de Luis Eduardo Garzón), se ha trabajado desde el programa ciudad-escuela-escuela-ciudad. A través de este plan de trabajo, se ha propuesto elevar el potencial educativo, cultural y productivo de la ciudad, buscando que ésta enseñe a través de sus actores, escenarios y prácticas, pero también de sus problemas y contradicciones ( Plan sectorial de educación, 2004-2008).

En las instituciones educativas oficiales se realizan en la jornada escolar expediciones formativas por la ciudad: sus instituciones, escenarios y eventos. Para ello, se establecieron convenios con las entidades distritales toma- das como escenarios educativos de la ciudad para ofrecer planes pedagógicos, tanto escolares como extra-escolares, dirigidos a sus estudiantes. El programa se desarrolla tanto en la jornada escolar, por medio de la salida de la escuela a los escenarios de la ciudad y la entrada de la ciudad a la escuela como tema de reflexión y aprendizaje, como en el tiempo de los alumnos por fuera de la escuela, para hacer un uso recreativo y formativo de la vida de la ciudad. Se promueve el uso lúdico y pedagógico de los escenarios urbanos en los ámbitos local y barrial mediante la conformación de grupos de interés en torno a las artes, la música, el cine, la danza, la literatura, la ciencia, la técnica, los oficios, el afianzamiento de conocimientos, la familia, y la vida productiva, social y politica (Cuervo y Páramo, 2005).

Ahora bien, desde los autores seleccionados en este estudio y en coherencia con los lineamientos de trabajo, se vio la necesidad, dentro de la investigación, de ahondar desde el Movimiento Ciudad Educadora en algunos de sus planteamientos generales. En ese orden se llega a la siguiente tabla:

Tabla 1. Autores y lineamientos

\begin{tabular}{ll} 
Autores & \multicolumn{1}{c}{ Lineamientos } \\
\hline Jaume Trilla Bernet & Educación integral, incluyente y permanente \\
Moacir Gadotti & Organización y participación de los sectores populares \\
Ramón Moncada & Formación de ciudadanía y los Derechos Humanos \\
Alejandro Álvarez & Derecho a la educación
\end{tabular}

Posteriormente, la investigación tomó la última de las subpreguntas del estudio, la cual se explicita en la tabla presentada a continuación, en la cual se plantean, desde los autores seleccionados, las diferentes aproxima- ciones metodológicas para poder trabajar más concretamente la relación de comunicación, educación y ciudadanía, desde el Movimiento Ciudad Educadora. 
Tabla 2. Autores y aproximaciones metodológicas

Autores

Jaume Trilla Bernet

Moacir Gadotti

Ramón Moncada

Alejandro Álvarez
Lineamientos

Cartografía social como base para la construcción de políticas y planes estratégicos.

Cartografía social. Mapa de las inclusiones y exclusiones. Empoderamiento de los sectores populares.

Construcción de políticas públicas. Trabajo intersectorial en formación de redes. Expediciones culturales en la ciudad.

Empoderamiento del docente en la construcción de ciudad. La ciudad como objeto de investigación.
Desde los anteriores resultados, se precisan en este orden de ideas varias reflexiones desarrolladas en la discusión de la investigación, que claramente muestra como desde el Movimiento Ciudad Educadora y los autores resaltados por esta investigación, es esencial obsenvar como tanto la academia como el gobierno (IDEP y Secretaría de Educación de Bogotá) deben trabajar conjuntamente hacia la construcción de políticas ciudadanas de orden participativo, donde la ciudad se convierta no en el objeto sino en un contexto que lleva a otros contextos, como la región y el país.

Es indispensable que, como lo plantea Álvarez, la investigación retome la ciudad a todo nivel, con la directriz de dar no sólo participación a los sectores culturales, sino responsabilidades a nivel de su labor en lo social. Se ve claramente cómo el trabajo que proponen Trilla y Gadotti de la cartografía social debe revisarse por parte de los grupos y líneas de investigación, para poder aportar a esta mirada esencial para la construcción social.

Se ven como ejes centrales en los resultados de esta investigación, igualmente, la importancia tanto de los derechos como de la inclusión, pero es importante también ahondar en los deberes del ciudadano, como parte central de esta inclusión y de la mirada de la participación anteriormente citada.
En ese orden de ideas, se proponen como aportes de esta investigación, no sólo los resultados del mismo, los cuales ayudan a dar un mapa de trabajo a desarrollar, sino una serie de preguntas que surgen del mismo. No es claro todavía el compromiso de la academia en este campo, lo cual implica que cada vez se deben desarrollar más no sólo espacios de debate gubernamental sino académico, que revisen estas posturas desde una críti$\mathrm{ca}$, que más que propender por una propuesta política, genere un macroproyecto prospectivo de trabajo conjunto.

Así las cosas, debe mirarse qué implica la región para esta mirada, y en ese orden la historia de lo público y lo privado en la ciudad y las repercusiones de estos ejes en la construcción de ciudadanía. De igual manera debe tenerse cautela con la construcción de políticas públicas, para no dejar los planteamientos del Movimiento Ciudad Educadora como un lineamiento de gobernantes, sino como una mirada crítica y una postura que debe revisarse y planificarse en los órdenes del otro, de las mismas problemáticas políticas y económicas del país. Sin olvidar que es importante acudir a la población para que participativamente pueda manifestar su percepción ante los procesos de ciudad educadora y escuela ciudad hasta ahora trabajados. 
En pocas palabras, se debe propender por una mirada donde la investigación y la participación sean centrales, hacia la construcción de país y ciudad, pero sin olvidar las importancia de posturas críticas que miren no solamente la acción sino el proceso y la prospectiva tanto de derechos como de deberes dentro de la organización social, y una participación con claros lineamientos y responsabilidades por parte de la ciudadanía y el mismo gobierno.

\section{Bibliografía}

Ayuntamiento de Barcelona (1990). I Congrés Internacional de Ciuts Educadores, Regidora d'Édicions I Publicacions. Barcelona.

Alderoqui, Silvia y Penchansky, Pompi (Comp.). Ciudad y ciudadanos. Aportes para la enseñanza del mundo urbano. Buenos Aires: Paidós, 2002. 240 p.

Barbero, Jesús Martín. "Comunicación y ciudad". En: Pensar la ciudad. Fabio Giraldo y Fernando Viviescas (Comp.). Bogotá: Tercer Mundo Editores, 1996, p. 45-79.

Cuervo, Mónica y Páramo, Pablo. (2005). Por publicar. Historia del espacio público en Bogotá durante el siglo XIX y su papel en la identidad de lugar del habitante de Bogotá: implicaciones para el movimiento de ciudad educadora. Universidad Pedagógica Nacional -Corporación Universitaria Iberoamericana.

Gómez-Granell Carmen y Vila Ignacio (Comp). La ciudad como proyecto educativo. Barcelona: Ediciones Octaedro. 2001. 159 p.

IDEP y Secretaría de Educación de Bogotá. Cátedra de pedagogía "Bogotá: una gran aula", 2004.

Moncada, Ramón. "La relación escuela entorno como entrada a la ciudad educadora". En: Revista Alegría de enseñar, № 44. Bogotá: Ministerio de Educación Nacional y Fundación FES, marzo de 2001, pp. $58-64$.

Moncada, Ramón y Toro, Javier. "Ciudad y escuela". En: La ciudad como escuela. Revista Educación y ciudad, № 2. Bogotá: Instituto para la investigación Educativa y el Desarrollo Pedagógico, IDEP, 1997. pp. 76-86.

Muntañola Thornberg, J. (1990). “La ciudad educadora desde la arquitectura". En: Ajuntament de Barcelona (1990) I Congrés Internacional de Ciuts Educadores, Regidora d'Édicions I Publicacions. Barcelona.

Prieto Castillo, Daniel. La comunicación en la educación. Medellín: Universidad Pontificia Bolivariana, Facultad de Comunicación Social. 2000, 207 p.

Trilla, Jaume. "La educacion y la ciudad". En: Otras educaciones. Universidad Pedagógica Nacional. Barcelona, España. 1993, pp. 177-203.

Villa, Marta Inés y Moncada, Ramón. Ciudad Educadora en Colombia. Corporación Región y La educación un propósito nacional. Bogotá, 1998, 147 p. 